\title{
A Soft Template Method to Graphene Oxide Nanosheet/Polypyrrole Nanofibers Hybrid as Supercapacitor Electrode Material
}

\author{
Weitong Xu, Xiwen Yang, Ke Jiang, Li Li Zhang ${ }^{2, ~ b}$, Lijuan Luo, Xiaoning \\ $\operatorname{Tian}^{1, a^{*}}$
}

${ }^{1}$ Department of Chemical Engineering, Ningbo University of Technology, Ningbo 315016, Zhejiang,

${ }^{2}$ Institute of Chemical and Engineering Sciences, A*STAR, 1 Pesek Road,Jurong Island 627833, Si ngapore

$\mathrm{a}^{\star}$ email : boxertxn@163.com, ${ }^{\mathrm{b}}$ lilizhang@gmail.com

Keywords: Graphene Oxide Nanosheet; Polypyrrole Nanofibers; Supercapacitor;

\begin{abstract}
A simple and effective method has been used to prepare graphene oxide and/or partially reduced graphene oxide-polypyrrole (GO/Ppy and PRGO/Ppy) composite with sandwich structures by using the soft template method. In the case of GO/Ppy8, a high specific capacitance of $456 \mathrm{~F} / \mathrm{g}$ at a current density of $2 \mathrm{~A} / \mathrm{g}$ and the capacitance retention ratio of about $68 \%$ when the current density increases from $300 \mathrm{~mA} / \mathrm{g}$ to $5 \mathrm{~A} / \mathrm{g}$ were obtained, indicating its potential for use as an electrode material for supercapacitors.
\end{abstract}

\section{Introduction}

Polypyrrole (Ppy) has been considered as one of the most promising conducting polymer electrode materials for supercapacitors because of its low cost, easy synthesis and relatively high conductivity. However, it suffers from a serious disadvantage of poor cycling stability during the charge-discharge process due to the swelling and shrinkage of the conducting polymer [1,2]. One way to overcome this disadvantage is using a substrate, which serves as a support material for the electrochemical utilization of Ppy and also provides the path for electron transfer. As a good support substrate graphene oxide (GO) has attracted great interest recently [3,4]. Herein, we demonstrate the coating of polypyrrole (Ppy) nanofibers on GO and partially reduced graphene oxide (PRGO) using an in situ polymerization method with the help of soft template, aiming to achieve the 3D layered structure, which can enhance the mechanical strength of the composite and stabilize the polymers during the charge/discharge process.

\section{Experimental}

\section{Materials preparation}

\section{Preparation of graphene oxide (GO)and partially reduced graphene oxide (PRGO)}

Graphite oxide was synthesized from natural graphite using a modified Hummers method [5].

Chemical conversion of graphene oxide to partially reduced graphene oxide (PRGO) was done according to the reported method [6].

\section{The coating of polypyrrole nanofibers onto $\mathrm{GO}$}

Polypyrrole nanofibers were coated on to GO sheets by using a soft template method according to our previous work [7]. Cetyltrimethyl-ammonium bromide (CTAB) was chosen as the soft template. $3.6 \mathrm{~g}$ of cetyltrimethyl-ammonium bromide (CTAB, 99\% Aldrich) dissolved in $40 \mathrm{~mL}$ of $1 \mathrm{M} \mathrm{HNO}_{3}$ solution was added to GO dispersion under stirring. Then, pyrrole (98\%, Aldrich) was added to the system at $10{ }^{\circ} \mathrm{C}$. After stirring for $60 \mathrm{~min}$, ammonium persulfate (APS, molar ratio of APS to pyrrole was 1:1) was added to initiate the polymerization. After $180 \mathrm{~min}$, the solid product was collected by filtration. The weight feed ratio of pyrrole to GO was varied as 8:1, 5:1, 2:1, and the resultant composites were named GO/Ppy8, GO/Ppy5, and GO/Ppy2, respectively. 


\section{The coating of polypyrrole nanofibers onto PRGO}

The coating of Ppy onto PRGO is similar to that of onto GO. The weight feed ratio of pyrrole to PRGO was varied as 8:1, 2:1, and the resultant composites were named PRGO/Ppy8 and PRGO/Ppy2, respectively.

\section{Characterization}

X-ray diffraction (XRD) patterns were collected on an XRD-6000 (Shimadzu, Japan) with Cu Ka radiation with $\lambda=0.15418 \mathrm{~nm}$. Fourier-transform infrared (FT-IR) spectra were acquired using the attenuated total reflectance (ATR) technique on an IRPrestige-21 (Shimadzu, Japan). The field-emission scanning electron microscope (FESEM) measurements were operated at $10 \mathrm{kV}$ (JSM 6700F, JEOL, Japan). And the transmission electron microscoe (TEM) measurements were conducted at $200 \mathrm{kV}(\mathrm{JEM} 2010$, JEOL, Japan).

\section{Electrochemical Measurement}

A three-electrode cell system was used to evaluate the electrochemical performance on an Autolab PGSTAT302N at room temperature. A $2 \mathrm{M} \mathrm{H}_{2} \mathrm{SO}_{4}$ aqueous solution was employed as the electrolyte. A platinum sheet and a saturated $\mathrm{Ag} / \mathrm{AgCl}$ electrode were used as the counter and the reference electrodes, respectively. The working electrode was prepared by casting a nafion-impregnated sample onto a glassy carbon electrode with diameter of $5 \mathrm{~mm}$.

\section{Results and Discussion}

\section{Morphology and Structure}

The morphology and structure of the Ppy, GO/Ppy, PRGO/Ppy and all composites are shown in Figures 1 and 2, respectively. For the pure Ppy sample prepared in the absence of GO or PRGO dispersion, only random Ppy fibers are seen as revealed by both the FESEM (Figure 1a) and TEM (Figure 2a ) images. The layered structure of the solid GO sample with stacked GO sheets can be seen from the FESEM image shown in Figure 1b. The TEM image (Figure 2b) showed a typical single layer GO sheet with a lateral dimension of several micrometers. As for the GO/Ppy composite, a flat and layered structure can be clearly seen from Figure 1 with Ppy fibers either sandwiched between GO sheets or on the GO surfaces. Thus, it can be concluded that polypyrrole nanofibers were successfully coated onto the GO sheets as we expected. From the image of GO/Ppy8 (Figure 1c), it is very easy for us to recognize which are the coated polypyrrole nanofibers and which are GO sheets as the white arrows pointed. The image of GO/Ppy5 (Figure 1g) shows a paper-like morphology, which is different from pure Ppy. It can be concluded that with the decrease of added monomer the nanofibers can still be coated onto GO sheets. However, with the further decrease of monomer amount the image of GO/Ppy2 (Figure 1e) shows no obvious polypyrrole nanofibers, and the stacked GO sheets can be found. This is because the amount of added monomer is too small to make the nanofibers coated onto GO sheets homogeneously. Therefore after desiccation process the GO sheets without coated polypyrrole nanofibers restacked together again. From the image of PRGO/Ppy8 (Figure 1d) we can clearly see both coated polypyrrole nanofibers and PRGO sheets. However, the coating of nanofibers is not as homogeneous as that of GO/Ppy. Because after the partially reduction process some of the surface oxygen functional groups on GO sheets was reduced, therefore we can assume that in the solution state the distribution of negative charge on PRGO sheets is not as homogeneous as that on GO sheets, which will affect the adsorption of positively charged soft template then lead to the unhomogenegous coating of nanofibers. Similar with GO/Ppy2, PRGO/Ppy2 (Figure 1f) also shows no obvious polypyrrole nanofibers due to the small amount of added pyrrole monomer.

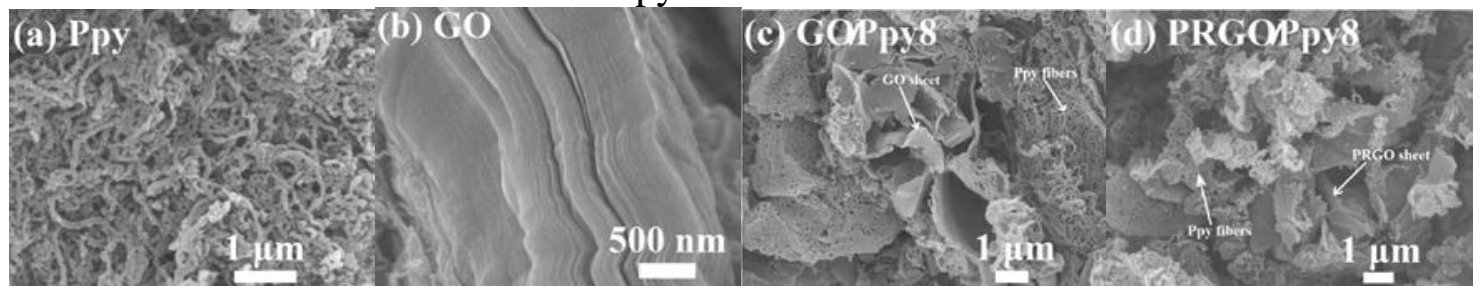




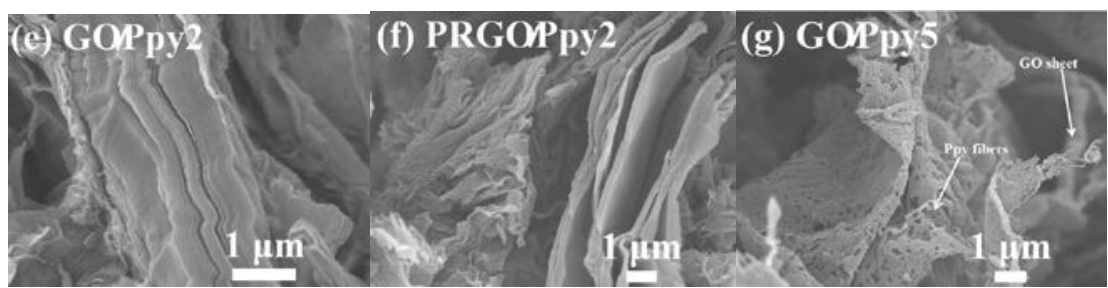

Figure 1. FESEM images of (a) Ppy, (b) GO, (c) GO/Ppy8, (d) PRGO/Ppy8, (e) GO/Ppy2, (f) PRGO/Ppy2, and (g) GO/Ppy5.

From the TEM image of GO/Ppy8 (Figure 2c) we can see that the paper-like GO sheet was homogeneously surrounded by polypyrrole nanofibers. Meanwhile, the polypyrrole fibers distributed both on the surface and between the PRGO sheets can be seen in Figure 2d. However, it can be seen that polypyrrole nanofibers coated onto GO sheets are more homogeneously than onto PRGO sheets, and this is in agreement with the FESEM results.

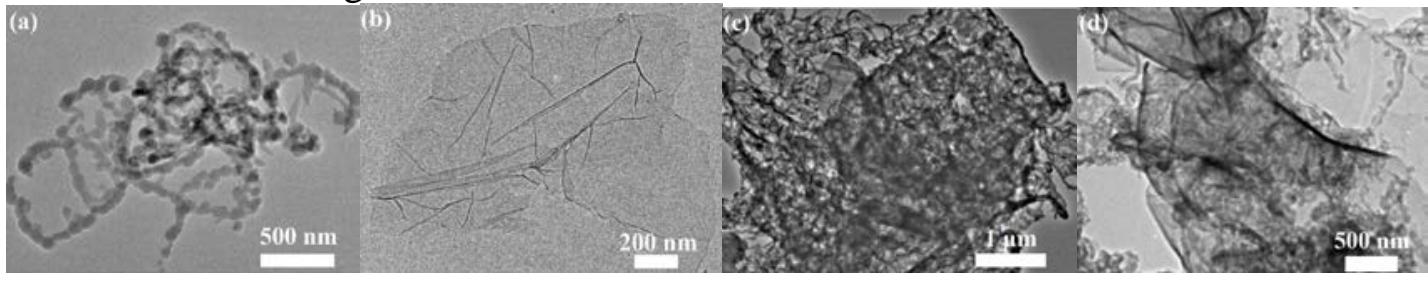

Figure 2. TEM images of (a) Ppy, (b) GO, (c) GO/Ppy8, and PRGO/Ppy8.

The structure of the composites was also investigated by powder X-ray diffraction (XRD) measurements. The XRD patterns of GO, PRGO, Ppy, GO/Ppy8, and PRGO/Ppy8 are shown in Figure 3. It can be seen from Figure 3A that GO sample after the drying process exhibited an intense, sharp peak centered at $2 \theta=11.6^{\circ}$, correlating to an interplanar spacing of $0.76 \mathrm{~nm}$ in the layer-like GO, which is larger than the d-spacing $(0.34 \mathrm{~nm})$ of pristine graphite $\left(2 \theta=26.5^{\circ}\right)$, as a result of introduction of oxygen functional groups [7-9]. This value can be assigned to the (001) reflection peak and might depend on the method of preparation and on the number of layers of water in the gallery space of the material. After partially reduction process, a broad reflection peak centered at $2 \theta=25.8^{\circ}$ was observed in the XRD pattern of PRGO (Figure 3A), which can be correlated to an interlayer spacing of $0.35 \mathrm{~nm}$ the graphene sample, indicating a loosely packed graphene sheets in PRGO [7-9], and it is different from the crystalline graphite. The pure Ppy sample exhibits the most intense peak at $2 \theta=25.5^{\circ}$ (i.e., $\mathrm{d}=0.31 \mathrm{~nm}$ ), which was ascribed to the planar aromatic pyrrole rings [2]. After the coating process, a new broad peak situated at around $23.4^{\circ}$ and 24.2 was found for GO/Ppy8 and PRGO/Ppy8, respectively, almost the same as that of pure Ppy as shown in Figure 3B.
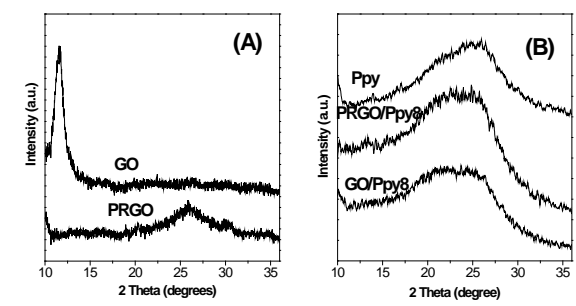

Figure 3. XRD patterns of (A) GO, PRGO; (B) Ppy, PRGO/Ppy8, and GO/Ppy8.

FTIR spectra of GO (Figure 4A) shows the characteristic absorption bands of oxide groups. The peak located at $1724 \mathrm{~cm}^{-1}$ is attributed to the $\mathrm{C}=\mathrm{O}$ stretching vibration. The bands at $3387 \mathrm{~cm}^{-1}$ and $1412 \mathrm{~cm}^{-1}$ were due to the vibration and deformation of $\mathrm{C}-\mathrm{OH}$ groups respectively. And the adsorption band at $1053 \mathrm{~cm}^{-1}$ can be assigned to C-O stretching [2,7,9-11]. It can be confirmed that through the chemical oxidization process the oxygen functional groups were attached on the GO sheets successfully. From Figure 4A we can see that the intensity of these absorption bands, however, are weakened for the partially reduced GO (PRGO), indicating a part of the oxygen groups had been removed by the reduction process. There are a number of new absorption bands that can be seen for sample GO/Ppy and PRGO/Ppy, which are similar with those of Ppy. The peaks located at 1548 and $1465 \mathrm{~cm}^{-1}$ are due to the fundamental stretching vibrations of pyrrole rings. The 
bands at 1034 and $1294 \mathrm{~cm}^{-1}$ are attributed to the $\mathrm{C}-\mathrm{H}$ deformation and $\mathrm{C}-\mathrm{N}$ stretching vibrations, respectively. The strong absorption band at about $1175 \mathrm{~cm}^{-1}$ indicates the doping state of Ppy $[2,7]$. All of the above peaks can be seen from sample Ppy, GO/Ppy and PRGO/Ppy, confirming the coating of Ppy onto GO and PRGO sheets. Additionally, no vibration signals due to methylene appearing at 2848 and $2929 \mathrm{~cm}^{-1}$ are seen, indicating that CTAB had been completely removed [7].
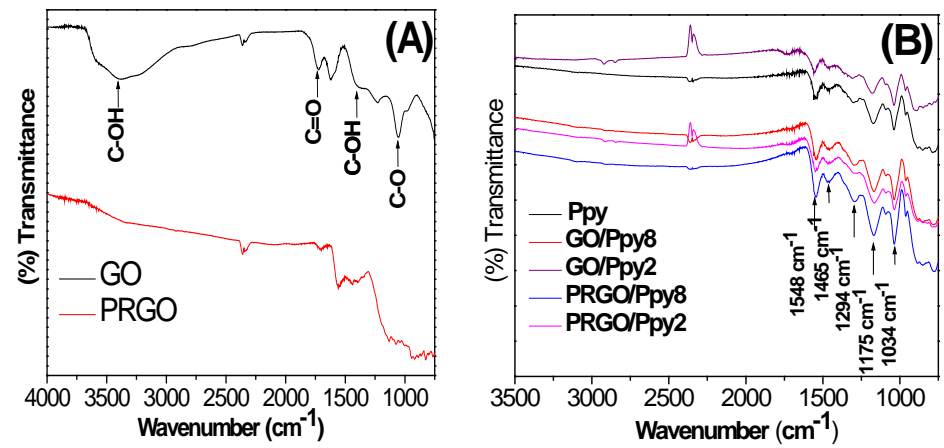

Figure 4. FTIR spectra of (A) GO, PRGO; (B) Ppy, GO/Ppy8, GO/Ppy2, PRGO/Ppy8 and PRGO/Ppy2.

\section{Electrochemical Properties of the Samples}

The cyclic voltammograms of the different composite electrodes with a potential window from -0.2 to $0.8 \mathrm{~V}$ (vs. Ag/AgCl) are shown in Figure 6. A rectangular shape of the cyclic voltammogram is expected for an ideal double layer capacitance behavior of an electrode. However, in the Ppy electrode, a deviation from such a rectangular shape is remarkable because of pseudofaradaic reactions of redox peaks as shown in Figure 6c. A region of reversible pseudofaradaic reaction of Ppy was observed with the oxidation peak at $+0.413 \mathrm{~V}$ and reduction peak at $+0.130 \mathrm{~V}$ in Figure $6 \mathrm{~d}$. Electrochemical capacitors built from the GO/Ppy8 and PRGO/Ppy8 exhibited a close to rectangular cyclic voltammograms at sweep rates ranging from 20 to $400 \mathrm{mV} \mathrm{s}^{-1}$ as shown in Figure $6 a$ and $b$, suggesting a highly reversible system. The cyclic voltammogram enclosed areas for the composites are: GO/Ppy8> PRGO/Ppy8>Ppy, demonstrating a much higher capacitance from the GO/Ppy8 as shown in Figure 6d. The behavior observed for the GO/Ppy8 is desirable in a capacitor electrode material.
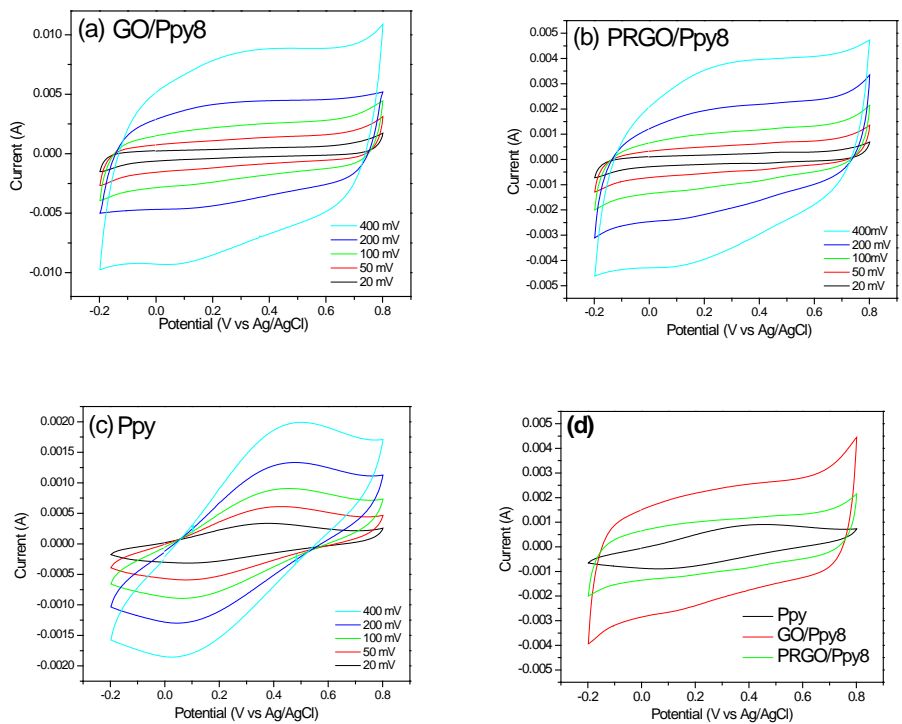

Figure 6. Cyclic voltammograms of (a) GO/Ppy8, (b) PRGO/Ppy8, (c) Ppy, The scan rate is changed from $20 \mathrm{mV} / \mathrm{s}$ to $400 \mathrm{mV} / \mathrm{s}$. (d)

$\mathrm{CV}$ profiles of different composite electrodes at a scan rate of $100 \mathrm{mV} / \mathrm{s}$.

The specific gravimetric capacitances of samples under different current loads are summarized in Table 1. After the coating of conducting polymer onto GO or PRGO composites show higher capacitance than that of pure PRGO due to the combination of electrical double layer capacitance and pseudocapacitance. 
Table 1. Specific gravimetric capacitance of various electrodes at different current densities.

\begin{tabular}{cccccc}
\hline Sample & $\mathbf{3 0 0} \mathbf{~ m A / g}$ & $\mathbf{5 0 0} \mathbf{~ m A / g}$ & $\mathbf{2 ~ A / g}$ & $\mathbf{3 ~ A / g}$ & $\mathbf{5 ~ A / g}$ \\
\hline PRGO & 124 & 98 & & & \\
GOPpy8 & 510 & 480 & 456 & 374 & 351 \\
GOPpy5 & 450 & 407 & 340 & 255 & 250 \\
GOPpy2 & 200 & 130 & 120 & 112 & 98 \\
PRGOPpy8 & 530 & 545 & 330 & 369 & 300 \\
PRGOPpy2 & 200 & 185 & 138 & 132 & 132 \\
Ppy & 360 & 250 & 150 & 120 & 130 \\
\hline
\end{tabular}

It can be obviously seen from Table 1 that the specific capacitance of GO/Ppy and PRGO/Ppy composites increased with the amount of coated polypyrrole, which implies that pseudocapacitance contribution from Ppy is very important to the higher specific capacitance. Moreover, when the current density increased from $300 \mathrm{~mA} / \mathrm{g}$ to $5 \mathrm{~A} / \mathrm{g}$, the capacitance retention ratio of Ppy is only $36 \%$. In contrast, the capacitance retention ratio for GO/Ppy8 and PRGO/Ppy8 is about $68 \%$ and $57 \%$ respectively, indicating a better rate capacity of the composites with respect to the single component of Ppy. The execllent capacitive performance of composite material would be attributed to the synergic integration between GO, PRGO and Ppy and include the following reasons: (1) the intercalated Ppy serving as effective spacers prevent GO or PRGO sheets from self-stacking and create critical spaces for free transport of electrolyte ions; (2) the high redox activity of Ppy offers significant pseudocapacitance; (3) the synergetic effects between GO, PRGO and Ppy, including the improvement of conductivity and the enhancement of ion diffusion by the formation of the $\pi-\pi$ networks between support substance and conducting polymer. The specific capacitance of composite materials increased with the increase of coated polypyrrole due to two main reasons. First, more polypyrrole can contribute more to material's pseudocapacitance. Secondly, the conductivity of composite materials was improved due to the higher loading of conducting polymer.

It is interested to see that PRGO/Ppy8 shows higher specific capacitance than that of GO/Ppy8 under the lower current density, however, the capacitance retention ratio of GO/Ppy8 is better than that of PRGO/Ppy8. From the preparation parameter we can see that the polymer amount ratio of GO/Ppy8 and PRGO/Ppy8 is similar. But, after the removal of oxygen-containing groups from the GO sheets, electronic structure and morphology of the GO will be changed. Ppy can not uniformly dispersed in the PRGO. Combine the above results it can be concluded that homogeneously coating of conducting polymer is very critical for synergetic effects between support material and coated conducting polymer. Although PRGO/Ppy2 and GO/Ppy2 exhibit the same specific capacitance under lower current density, specific capacitance of GO/Ppy2 decreased dramatically when the current density increased. As we mentioned above the polymer amount ratio of GO/Ppy2 and PRGO/Ppy2 is almost the same, which is too small to be coated onto the carbon sheets homogeneously. In this kind of situation it can be assumed that the electrical double layer capacitance is the predominant part of the whole capacitance. Because the conductivity of PRGO/Ppy2 is much higher than that of GO/Ppy2 due to the insulate character of GO, however, good conductivity is beneficial for the electrical double layer capacitance, therefore PRGO/Ppy2 shows better electrochemical property.

\section{Conclusions}

In conclusion, a simple and effective method has been demonstrated to prepare graphene oxide and/or partially reduced graphene oxide-polypyrrole (GO/Ppy and PRGO/Ppy) composite with sandwich structures by using the soft template method. It is found that the specific capacitance of GO/Ppy hybrid with a higher loading of Ppy is mainly attributed to pseudocapacitance, while those with smaller loading of Ppy exhibits electrical double layer capacitance. It can be concluded that homogeneously coating of conducting polymer is very critical for synergetic effects between support material and coated conducting polymer. 


\section{Acknowledgment}

This work was financially supported by the Chinese National Natural Science Foundation (No. 21306091), the Zhejiang Provincial Natural Science Foundation of China (No. LQ13E020002), the Ningbo Natural Science Foundation (No. 2012A610130), New talent plan of Zhejiang Province (No.2012R422020, No. 2014R422004).

\section{References}

[1] Hyunwoo Kim, Ahmed A. Abdala, and Christopher W. Macosko. Graphene/Polymer Nanocomposites [J].Macromolecules, 201043 6515-6530.

[2] Chengzhou Zhu, Junfeng Zhai, Dan Wen Shaojun Dong. Graphene oxide/polypyrrole nanocomposites: one-step electrochemical doping, coating and synergistic effect for energy storage [J]. Journal of Material Chemistry, 201222 6300-6306.

[3] Zhe-Fei Li, Hangyu Zhang, Qi Liu, Lili Sun, Lia Stanciu, Jian Xie. Fabrication of High-Surface-Area Graphene/Polyaniline Nanocomposites and Their Application in Supercapacitors [J]. ACS Applied Materials and Interfaces, 20135 2685-2691.

[4] Zhiping Song, Terrence Xu, Mikhail L. Gordin, Ying-Bing Jiang, In-Tae Bae, Qiangfeng Xiao, Hui Zhan, Jun Liu, Donghai Wang. Polymer-Graphene Nanocomposites as Ultrafast-Charge and -Discharge Cathodes for Rechargeable Lithium Batteries [J]. Nano Letters, 201212 2205-2211.

[5] William S. Hummers Jr., Richard E. Offeman. Preparation of Graphitic Oxide [J]. Journal of American Chemical Society, 195880 (6) 1339-1339.

[6]Sasha Stankovich, Dmitriy A. Dikin, Richard D. Piner, Kevin A. Kohlhaas, Alfred Kleinhammes, Yuanyuan Jia, Yue Wu, SonBinh T. Nguyen, Rodney S. Ruoff. Synthesis of graphene-based nanosheets via chemical reduction of exfoliated graphite oxide [J]. Carbon, 2007 45, 1558-1565.

[7] Li Li Zhang, Shanyu Zhao, Xiao Ning Tian, X.S. Zhao. Layered Graphene Oxide Nanostructures with Sandwiched Conducting Polymers as Supercapacitor Electrodes [J]. Langmuir, 201026 17624-17628.

[8] Kai Zhang, Li Li Zhang, X.S. Zhao, Jishan Wu. Chem. Graphene/Polyaniline Nanofiber Composites as Supercapacitor Electrodes [J]. Chemistry of Materials, 201022 1392-1401.

[9] Zhongqing Jiang, Yilei Shi, Zhong-Jie Jiang, Xiaoning Tian, Lijuan Luo Weiheng Chen. High performance of a free-standing sulfonic acid functionalized holey graphene oxide paper as a proton conducting polymer electrolyte for airbreathing direct methanol fuel cells [J]. Journal of Material Chemistry, A 20142 6494-6503.

[10] Zhongqing Jiang, Zhong-jie Jiang, Xiaoning Tian and Weiheng Chen. Amine-functionalized holey graphene as a highly active metal-free catalyst for the oxygen reduction reaction [J]. Journal of Material Chemistry A, 20142 , 441-450.

[11] Zhongqing Jiang, Bo Pei and Arumugam Manthiram. Randomly stacked holey graphene anodes for lithium ion batteries with enhanced electrochemical performance [J]. Journal of Material Chemistry A, 20131 7775-7781. 